\title{
L'analyse réflexive au service de l'émancipation : construire des compétences et se construire
}

Valérie SOUBRE, ILCF (Institut de Langue et de Culture Française), Université Catholique de Lyon / secteur langues du GFEN (Groupe français d'Education Nouvelle), France

\section{Introduction}

Les travaux du Conseil de l'Europe ont abouti à l'élaboration du Cadre européen commun de référence pour les langues (désormais CECRL), dont le sous-titre est «Apprendre - Enseigner - Evaluer». Dans le CECRL, est explicité ce que recoupent les différents types et fonctions de l'évaluation, que ce soit l'évaluation diagnostique, sommative, certificative ou formative; il y est toutefois précisé que «les enseignants deviennent de plus en plus responsables de l'évaluation formative autant que sommative de leurs élèves à tous niveaux » (22). Aujourd'hui, les pratiques de l'évaluation dans les classes articulent ces différentes dimensions : à la fois attester des acquis mais aussi faire « de plus en plus appel à l'auto-évaluation des apprenants, que ce soit pour organiser et planifier leur apprentissage ou pour rendre compte de leur capacité à communiquer dans une langue [...]»

L'évaluation formative fait l'objet d'un large consensus au niveau conceptuel, toutefois dans le cadre concret des classes de français langue étrangère, ses pratiques sont peu répandues. Pourtant de nombreux théoriciens tels que Philippe Perrenoud posent la problématique de l'apprentissage pour l'apprenant en tant qu'espace où s'investir et s'impliquer :

Apprendre est une activité fort capricieuse, qui exige une forte implication de la personne, qui doit donc avoir du sens, si possible au-delà de la simple conformité aux attentes de l'autre. Plus les apprentissages sont de haut niveau taxonomique, plus ils s'étalent dans le temps, passent pas des constructions et reconstructions partiellement invisibles et imprévisibles. Toute perspective constructiviste et interactionniste le réaffirme : c'est l'élève qui apprend, à son rythme, en suivant ses propres modes de pensée. Les enseignants ne peuvent qu'aménager des situations didactiques, en espérant qu'elles seront fécondes, qu'elles se présenteront au bon moment, que l'élève voudra et pourra s'y investir. (Pédagogie, 119-128)

Perrenoud montre donc ici que l'apprenant est acteur de son apprentissage, l'enseignant créant quant à lui, les situations les plus favorables possibles à cette mise en activité. 
Adopter cette conception socioconstructiviste de l'apprentissage, c'est-à-dire placer l'apprenant au centre du processus d'enseignement apprentissage afin qu'il construise, avec ses pairs, ses compétences, réinterroge les fonctions de l'évaluation formative. Dans cet article, nous allons nous y intéresser en tant qu'espace à construire avec les apprenants. Cet espace que nous nommerons « agir évaluatif partagé » (Jorro) permettra de souligner le rôle de l'analyse réflexive et comment celle-ci est au service de l'émancipation. Nous interpellerons tout d'abord les théories et les pratiques enseignantes de l'évaluation formative. Puis en analysant les ressources et les contraintes et en proposant une définition activée et opérationnelle, nous montrerons que l'analyse réflexive permet aux élèves, tout en articulant objectifs de formation, activités et positionnement ou validation, de se donner en permanence une vision moins parcellaire du parcours de formation. Pour finir, nous questionnerons cette vision globale des apprentissages construite par l'apprenant, qui progresse dans son apprentissage en se «tissant » un réseau de savoirs, de savoir-faire et de savoir-être organisés et structurés.

\section{Théories et pratiques des enseignants}

La motivation des élèves à apprendre est devenue un enjeu majeur du débat sur l'apprentissage. Dans cette optique, les conceptions de la pédagogie dite de la réussite veulent privilégier les situations d'apprentissage à fonction formative plutôt que normative. L'évaluation formative a pour but de guider l'élève, lui permettre de reconnaître, de comprendre et de corriger lui-même ses erreurs (fonction corrective), de l'informer sur les étapes franchies ou non, tout en informant l'enseignant sur les effets réels de son action pédagogique (fonction régulatrice) ; et en particulier, comme le souligne également Jacques Weiss sur une évolution du paradigme de l'évaluation-sanction vers celui de l'évaluationrégulation.

Il ne s'agit donc plus d'évaluer un élève, ponctuellement par rapport à la seule norme, mais de lui permettre de mettre en évidence ses réussites et ses difficultés, tout au long de son apprentissage, pour qu'il puisse y remédier, tout en lui reconnaissant son caractère singulier.

Par ailleurs, Weiss affirme que la réussite de l'élève, et donc sa motivation, sont liées aux pratiques correctrices des enseignants. Il ajoute que la difficulté de l'évaluation vient du fait qu'élève et évaluateur ne donnent pas nécessairement le même sens à une tâche, et que l'évaluation formative vise à réaliser ce recouvrement en permettant à l'élève de s'approprier les critères d'évaluation et les moyens à mettre en œuvre pour s'y conformer avec succès en 
se dégageant de l'implicite. Ainsi, ce type d'évaluation apparaît comme un outil d'apprentissage, qui vise à rendre l'élève acteur de son apprentissage.

Toutefois, dans les classes de français langue étrangère, il est souvent difficile pour les apprenants étrangers de se situer dans leur apprentissage, notamment parce que malgré l'intérêt porté par les acteurs à l'évaluation formative, dans les pratiques de classe, celle-ci apparait rarement de manière formalisée, les enseignants la jugeant trop complexe et chronophage. Cette représentation de l'évaluation formative est souvent associée à l'idée que les apprenants ne savent pas s'auto-évaluer et donc qu'il leur est très difficile de se positionner par rapport à leurs apprentissages et d'en parler de manière juste.

De ce fait, les apprenants dépendent quasi uniquement de l'enseignant pour leur évaluation et donc leur progression. Ils n'ont alors que très peu de visibilité sur leur parcours de formation. Cet état de fait amène les apprenants à situer leurs acquis lors des évaluations sommatives, ce qui pose une question importante : à quelle fonction de l'évaluation se réfèret-on donc dans cette situation?

Néanmoins, il est à souligner que certains enseignants intègrent dans leurs pratiques l'auto-évaluation, mais la plupart du temps, elle est mise en place pour évaluer une activité langagière (par exemple: la production orale) ou bien sur un savoir-faire et non sur l'ensemble. Il est plus rare qu'elle concerne des savoirs.

Cela est dommageable quand on sait que l'évaluation formative, et notamment l'autoévaluation, par le travail de retour sur ce qui a été fait dans des phases de métacognition, est considérée comme un facteur favorable à l'apprentissage et au transfert. En d'autres termes, exercer une activité réflexive sur sa cognition permet à l'élève d'en améliorer l'efficacité (Wolfs, Romainville, Noël).

A ce sujet, nous pouvons nous demander en quoi et comment la mise en place d'une pratique réflexive permet de générer des effets positifs sur l'enseignement - apprentissage. Du point de vue de l'enseignant, accompagner lors des situations d'apprentissage de l'autoévaluation nécessite de reconnaître que cette compétence est en devenir et que l'on parle de processus d'auto-évaluation (entre ne pas savoir s'auto-évaluer et le faire, il y a différents degrés de maîtrise). A la lumière des travaux de Lev Vygotsky, l'accompagnement effectué par l'enseignant est envisagé comme une aide, un guide qui permet d'aller plus loin. Les savoirs ne peuvent se construire que dans un système d'interactions complexes, comprenant celles entre enseignant et apprenant ou entre pairs.

En conséquence, accompagner l'élève dans sa démarche d'auto-évaluation s'inscrit dans ce que Jérôme Bruner appelle «l'interaction de tutelle », où l'apprenant intervient sur 
les conditions mêmes de son apprentissage par le mode de communication qu'il instaure avec l'enseignant. Il dénonce ainsi l'hypothèse implicite que «celui qui apprend est seul et sans aide ». Cette interaction trouve sa complémentarité dans les processus d'interaction entre pairs, qui sont au moins aussi importants que ceux qui se déroulent avec l'enseignant. C'est ainsi que les outils, permettant de faire le point, de revenir sur ce qui a été fait, sur ce qui a été réussi ou non, trouvent leur place dans l'enseignement apprentissage du français langue étrangère.

Une fois ces constats faits, l'apprentissage comme une co-construction nécessite un nouvel espace à créer : l'agir évaluatif partagé.

Dans la mise en place de l'auto-évaluation en classe, les enseignants peuvent parfois être tentés d'en rester à ces injonctions «Soyez autonomes, les élèves et auto-évaluez-vous » et donc de travailler des situations d'auto-évaluation sans les inscrire dans un processus d'évaluation formative visant l'autonomisation. Dans ce cas, il n'y a pas de processus mis en place. Ainsi, que faudrait-il mettre en œuvre pour rendre cette auto-évaluation efficiente pour les apprentissages des apprenants ?

L'analyse réflexive peut tout d'abord être définie dans un agir évaluatif partagé entre l'enseignant et les apprenants. L'enseignant doit alors mettre en place une culture commune de l'évaluation formative, tant au niveau des conceptions qu'au niveau des situations vécues en classe.

Dans la démarche socioconstructiviste, la norme d'évaluation fait conflit avant d'être régulée pour devenir un référent utile. Pour cela, l'enseignant propose des situations dans lesquelles les élèves peuvent confronter leur propre norme, leurs représentations et ainsi verbaliser puis conscientiser. Ce travail de confrontation se fait dans une inter-évaluation (entre élèves), qui peut avoir comme point de départ une auto-évaluation (l'élève vis-à-vis de lui-même). Le passage de l' «inter» au «socio» permettrait la construction d'un agir évaluatif partagé, c'est-à-dire des valeurs, des habitus, des postures et une norme en commun.

\section{Définir l'analyse réflexive...}

L'analyse réflexive comme élément de l'agir évaluatif partagé pourrait permettre à l'enseignant de mieux enseigner, c'est-à-dire d'améliorer sa compétence professionnelle et à l'élève de mieux apprendre, c'est-à-dire d'améliorer ses apprentissages (Médioni).

L'analyse réflexive est la conscientisation des processus d'apprentissage par l'élève lui-même. Elle nécessite de se construire un comportement cognitif afin de récapituler les procédures et les processus qui ont permis d'apprendre (Médioni). 
L'analyse réflexive est un élément indissociable de toute démarche d'enseignementapprentissage d'une langue. En interpellant les théories et les pratiques enseignantes de l'évaluation formative, en analysant les ressources et les contraintes et en proposant une définition activée et opérationnelle, nous souhaitons montrer qu'elle permet aux élèves, tout en articulant objectifs de formation, activités ou expériences de formation et positionnement ou validation, de se donner en permanence une vision moins parcellaire du parcours de formation, tout en se «tissant» un réseau de savoirs, de savoir-faire et de savoir-être organisés et structurés.

Associer les apprenants, c'est leur présenter clairement en début d'année les objectifs ; au cours de l'année, à chaque début de séquence, l'objectif est également annoncé et expliqué aux élèves ; en fin de séquence, la classe fait un bilan de ce qu'elle a appris, et aide ainsi chaque élève à se positionner en fonction de ses apprentissages. Scallon (Apprentissages) insiste fortement sur cette démarche d'auto-évaluation : «C'est une habileté à développer en parallèle avec les objectifs d'apprentissage qui sont l'objet du portfolio. L'élève s'auto-évalue lorsqu'il se sert de ses réalisations pour témoigner de sa progression, lorsqu'il fait état des difficultés qu'il a éprouvées et des moyens qu'il a adoptés pour les surmonter. »

Si l'auto-évaluation et l'analyse réflexive visent à l'autonomisation de l'apprenant dans ses apprentissages, cela signifie qu'un apprenant expert peut mobiliser lui-même les moyens nécessaires à la réalisation d'une tâche donnée. Cela nécessite qu'il soit capable de se distancier par rapport à son activité, pour surveiller ce qu'il fait et si nécessaire s'autoréguler. Il doit être capable de prendre conscience de sa manière d'apprendre et d'exercer un contrôle sur celle-ci. Autrement dit, il doit mettre en œuvre des stratégies métacognitives, que Anne-Marie Doly définit comme « une distanciation réflexive du sujet qui fait et qui surveille ce qu'il fait pour s'auto-évaluer et s'auto-réguler en utilisant en particulier les connaissances qu'il a de ses propres cheminements [...]». D'autant que la métacognition est, pour Doly, nécessaire à l'effort d'apprentissage : avoir conscience de son pouvoir de contrôle et de maîtrise de sa pensée permet de développer un concept positif de soi-même, qui rend plus entreprenant et audacieux. De ce fait, on contribue également à développer chez les apprenants des capacités d'analyse réflexive.

L'analyse réflexive doit également permettre l'auto-évaluation par l'apprenant, soit par évaluation comparative du même objet à deux moments différents (par exemple, un état des savoirs, ou une manière de réaliser une tâche), soit par la co-évaluation. Les exemples ciaprès sont issus du travail de classe en français langue étrangère dans le cadre de la mise en 
place d'un portfolio ${ }^{1}$. Lors d'une compréhension écrite, l'élève note les stratégies qu'il mobilise. Il en fait de même lors de la deuxième compréhension écrite. Par comparaison évaluative, il peut s'interroger sur l'évolution de sa manière de mobiliser des stratégies. Deux apprenants peuvent également comparer leur manière de faire, en utilisant les traces de leur portfolio. Toutefois, suite à l'observation des élèves, nous avons pu remarquer qu'ils ont des difficultés à utiliser les traces de leur activité : ils ont tendance à penser que ce qui est fait n'est plus utile et qu'ils ne peuvent pas exercer de contrôle sur leur activité. L'enseignant propose alors des situations pédagogiques qui permettent aux élèves de faire des liens entre production finale et démarche suivie. Par ailleurs, l'élève devient responsable lorsqu'il assume ce qu'il fait, lorsqu'il est capable d'en parler aux autres, d'expliquer ses démarches et ses choix. Lorsqu'en contrepartie, il accepte d'apprendre des autres, ce processus réflexif permet de l'engager dans une autonomisation dans ses apprentissages, pouvant aller jusqu'à la transgression positive des situations et des normes de la classe.

\section{L'analyse réflexive permettant l'autonomie et l'émancipation...}

De fait, progressivement, au fur et à mesure des situations de classe, les élèves parviennent à utiliser opportunément des expériences passées dans des situations nouvelles. Qui plus est, la mise en œuvre de l'analyse réflexive manifeste une appropriation de l'acte de l'évaluation par l'élève, et le fait accéder à une certaine autonomie, voire émancipation vis-à-vis du savoir et de l'enseignant.

Lors de l'observation citée ci-dessus des apprenants en situation d'analyse réflexive, celle-ci s'est basée sur des indicateurs précis permettant d'observer des comportements, des attitudes, des savoir-être. La définition de ces items s'est appuyée sur la taxonomie de Benjamin Bloom. Bloom montre que, dans le processus d'apprentissage, les capacités peuvent être mesurées sur une échelle allant des opérations mentales les plus simples aux plus complexes, révélant ainsi que le plus haut niveau de maîtrise est l'évaluation, qui est l'objectif final de la démarche portfolio, c'est-à-dire la capacité à mener une analyse réflexive pertinente tout au long de son parcours d'apprentissage. Par ailleurs, pour se représenter l'apprentissage, l'élève doit pouvoir identifier les usages qu'il pourra faire des résultats de cet apprentissage, que ce soit au niveau personnel ou professionnel. Il doit pouvoir faire des liens entre les situations d'apprentissage, son projet personnel, son projet professionnel. Autrement dit, il doit pouvoir répondre à la question «A quoi va me servir cet apprentissage ? ». C'est à cette condition que l'élève se mobilise sur la tâche. Il est donc nécessaire de lui laisser le temps de se confronter à la tâche, pour qu'il clarifie les objectifs, ceux de l'enseignant, et les 
siens personnels, par une verbalisation appropriée. Cette fonction de l'analyse réflexive consiste donc en la projection des apprentissages : l'élève réfléchit aux situations dans lesquelles il pourra transférer les compétences qu'il développe, et ainsi se mobiliser sur son apprentissage. Il peut les lister, comme autant de situations à observer par la suite, autour de lui, pour améliorer ses compétences. L'enseignant peut utiliser les images mentales que l'élève se projette, pour le faire réfléchir à des critères d'évaluation de l'acquisition de cette compétence. Il est donc nécessaire de penser l'existence d'une zone de développement potentiel qui, moyennant diverses formes de médiation, favorise l'accès à un stade supérieur. Vygotsky, par le concept de zone proximale de développement (voir Schneuwly), indique que c'est le développement qui peut tirer l'apprentissage vers l'avant. En outre, en donnant à voir le parcours individuel, l'analyse réflexive permet à l'enseignant de suivre la progression de chacun et de le guider, de l'évaluer, de le faire réfléchir. Après l'activité, l'élève va pouvoir réfléchir et analyser des questions telles que : Qu'ai-je fait? Ai-je réussi ou pas à faire ? Dans ce que j'ai fait, ce qui m'a permis de réussir ou non ? Comment aurais-je pu faire autrement?

Cette évaluation partagée impose à l'élève de se situer par rapport à son apprentissage sans forcément dépendre du retour de l'enseignant. Pour l'élève, se positionner vis-à-vis de ses apprentissages permet aussi de se positionner vis-à-vis de son parcours de formation et ainsi d'en être beaucoup plus maître d'œuvre. Les processus mis en œuvre engendrent tout aussi bien de la normalisation que de la transgression, suivant les situations, la tension entre les deux étant constitutive de l'autonomisation. Cette autonomisation activée peut alors produire une émancipation, envisagée comme une coconstruction entre une personne et des situations et comme un mouvement en avant où se situe un nouvel espace à créer.

Les démarches intégrant l'analyse réflexive permettent donc une plus grande explicitation des objectifs, une plus grande cohérence dans les situations d'apprentissage et demandent plus de souplesse dans la gestion de la planification sur l'année, ainsi qu'un réel partage concernant les critères d'évaluation.

Pour Perrenoud, il y a alors changement du contrat didactique :

Dans une pédagogie centrée sur les savoirs, le contrat de l'élève est d'écouter, de tenter de comprendre, de faire consciencieusement ses exercices et de restituer ses acquis dans le cadre de tests de connaissance papier-crayon, le plus souvent individuels et notés. Dans une approche par compétence, le rôle de l'élève est de s'impliquer, de participer à un effort collectif pour réaliser un projet et construire, par la même occasion, de nouvelles compétences. Il a droit aux essais et aux erreurs. Il est invité à faire part de ses doutes, à expliciter ses raisonnements, à prendre conscience de ses façons de comprendre, de 
mémoriser, de communiquer. On lui demande en quelque sorte, dans le cadre de son métier d'élève, de devenir un praticien réflexif. On l'invite à un exercice constant de métacognition et de métacommunication. Un tel contrat exige davantage de cohérence et de continuité d'une classe à la suivante et un effort incessant d'explicitation et d'ajustement des règles du jeu. («Savoirs », 6-10)

Ainsi, sans cette phase de mise à distance que permet l'auto-évaluation, le processus d'apprentissage reste inachevé. Les savoirs, savoir-faire et savoir-être mis en œuvre doivent être nommés et décrits pour être pleinement repérés et devenir mobilisables dans d'autres situations.

Comme le rappelle Christian Puren,

Si l'un des objectifs premiers de l'enseignement scolaire des langues est désormais la formation à l'apprentissage, l'un des aspects essentiels de l'évaluation dans ce contexte est donc clairement la formation à l'autoévaluation, qui ne va pas sans poser de multiples problèmes, mais qui constituera sans aucun doute l'un des grands enjeux de l'évolution de la didactique scolaire dans les années à venir. («Problématique »,29)

Si les apprenants disposent d'occasions pour travailler entre eux leur évaluation, ils consolident mutuellement leurs points forts et agissent sur leurs points faibles. L'explicitation des critères d'évaluation leur permet de connaître ce que l'enseignant attend d'eux. Un apprenant entreprend plus ou moins rapidement de s'auto-évaluer, c'est-à-dire d'utiliser les critères sur son propre travail. L'aider à entrer dans une démarche d'analyse réflexive, c'est alors lui permettre d'apprendre à s'auto-évaluer et de manifester par cela, au fur et à mesure du travail, son degré d'autonomie. Pour conclure sur ce point, Anne Jorro affirme «à chaque élève de devenir un auto-évaluateur, sans quoi l'émancipation serait vaine. » La question est alors de se libérer de la tutelle unique de l'enseignant évaluateur : «en dénouant la relation évaluateur/évalué, le sujet émancipé peut réinjecter dans l'action, ce processus évaluatif. Porté par des gestes qui ont pris sens pour lui, l'élève investit la posture d'évaluateur. »

\section{Pour conclure...}

Auto-évaluation, analyse réflexive, les enseignants de français langue étrangère mettent en œuvre de plus en plus fréquemment des démarches réflexives chez leurs élèves. Ceci génère des évolutions fortes : d'une part dans le processus d'apprentissage d'une langue étrangère en aidant l'apprenant à mieux se situer dans son apprentissage et d'autre part, dans le processus d'enseignement en donnant à l'enseignant des informations relatives aux acquisitions des élèves. 
La mise en ouvre de l'évaluation formative via les analyses réflexives redéfinit le contrat d'évaluation dans la classe. En effet, nous pouvons affirmer qu'avec une telle démarche nous pouvons dépasser les difficultés à penser l'évaluation autrement que dans sa dimension sociale, nous avons réuni un certain nombre de conditions permettant à la fois la formation à l'autonomie, la prise en compte de toutes les expériences et acquis, ainsi que leur valorisation. Pour finir, nous avons ouvert à une autre culture de l'évaluation au service de l'élève et de ses apprentissages, à côté de l'évaluation sociale.

Force est de constater que, tout en articulant objectifs de formation, activités ou expériences de formation et positionnement ou validation, l'analyse réflexive permet aux élèves de se donner en permanence une vision moins parcellaire du parcours de formation, par la création d'un réseau de savoirs, de savoir-faire et de savoir-être organisés et structurés. De fait, progressivement les élèves parviennent à utiliser opportunément des expériences passées dans des situations nouvelles; ceci manifeste bien ici une appropriation de l'acte de l'évaluation par l'élève.

Ainsi, à côté de l'évaluation à dominante sociale (note, bulletins, appréciations...), il y a l'ouverture à une culture de l'évaluation au service de l'élève et de ses apprentissages par une appropriation progressive de ses ressources (descripteurs, critères, activité métacognitive).

Pour terminer sur le thème de la transgression, nous souhaiterions citer Perrenoud («Savoirs », 33) : Qu'est-ce qu'un praticien réflexif ? C'est fondamentalement quelqu'un qui transgresse ou conteste les interdits (par exemple: demander pourquoi, envisager des alternatives, mettre en débat ce qui va de soi dans les écoles).

\section{Bibliographie}

Allal, Linda. «Régulations méta cognitives : quelle place pour l'élève dans l'évaluation formative ?» Evaluation formative et didactique du français: 3. Dir. Allal Linda, Daniel Bain et Philippe Perrenoud. Paris : Delachaux et Niestlé, 1993. 81-98.

Bloom, Benjamin. Taxonomie des objectifs, tome 1, Domaine cognitif. Montréal : Education nouvelle, 1969.

Bruner, Jérôme. Le développement de l'enfant : savoir faire, savoir dire. Paris : PUF, 1987.

Conseil de l'Europe. Cadre européen commun de référence pour les langues. Paris : Didier, 2001.

Doly, Anne-Marie. «La métacognition pour apprendre à l'école ». Les cahiers pédagogiques 381 (2000). 
Donnay, Jean et Evelyne Charlier. Apprendre par l'analyse de pratiques : initiation au compagnonnage réflexif. Namur : PU de Namur, 2006.

GFEN. Réussir en langues. Un savoir à construire. Lyon : Chronique Sociale, coll. Pédagogie et formation, 2002.

Grangeat, Michel. La métacognition, une aide au travail des élèves, Paris : ESF, coll. Pédagogies, 1997.

Huver, Emmanuelle et Claude Springer. L'évaluation en langues, Paris: Didier, coll. Langues et didactiques, 2011.

Jorro, Anne. L'enseignant et l'évaluation. Des gestes évaluatifs en question, Bruxelles : De Boeck, coll. Pratiques pédagogiques, 2000.

Médioni, Marie-Alice. 25 pratiques pour enseigner les langues. GFEN, Chronique Sociale, 2010.

Noel, Bernadette, Michel Romainville et José Luis Wolf. « La métacognition : facettes et pertinence du concept en éducation ». Revue française de pédagogie 112 (1995).

Perrenoud, Philippe. Développer la pratique réflexive dans le métier d'enseignant. Professionnalisation et raison pédagogique. Paris : ESF, 2001.

---. «Des savoirs aux compétences : les incidences sur le métier d'enseignant et sur le métier d'élève ». Pédagogie collégiale $\quad 9: \quad 2$ (1995): 6-10. (http://www.unige.ch/fapse/SSE/teachers/perrenoud/)

---. La pédagogie à l'école des différences. Paris : ESF, 1995 (1992).

Puren, Christian. La didactique des langues et des cultures à la croisée des méthodes. Essai sur l'éclectisme, Paris : Didier, coll. CREDIF Essais, 1994.

---. «La problématique de l'évaluation en didactique scolaire des langues ». Les langues modernes 2 (2001) : 12-29.

Saint Pierre, Lise. «L'habileté d'auto-évaluation: comment la développer?» $\mathrm{U}$ de Sherbrooke, Performa, 2004. www.cdc.qc.ca

Scallon, Gérard. L'évaluation formative, Bruxelles : De Boeck, coll. Pratiques pédagogiques, 2000.

---. L'évaluation des apprentissages dans une approche par compétences, Bruxelles : De Boeck, coll. Pratiques en développement, 2004.

Schneuwly, Bernard et Jean-Paul Bronckart. Vygotsky aujourd'hui. Paris : Delachaux et Niestlé, 1985.

Vygotsky, Lev. Pensée et langage. Paris : Messidor / Editions sociales. 1985. 
Weiss, Jacques. «Le portfolio, instrument de légitimation et de formation ». Revue Française de Pédagogie 132 (2000).

\section{NOTE}

${ }^{1} \mathrm{http}: / /$ www.coe.int///dg4/education/elp/default_FR.asp : Le Portfolio européen des langues (PEL) a été mis au point par l'Unité des politiques linguistiques du Conseil de l'Europe pour

- contribuer au développement de l'autonomie de l'apprenant, du plurilinguisme ainsi que de la sensibilité et de la compétence interculturelle

- permettre aux utilisateurs de consigner les résultats de leur apprentissage linguistique ainsi que leur expérience d'apprentissage et d'utilisation de langues. 